\title{
Escrita científica: conhecimento dos acadêmicos de enfermagem
}

\author{
Scientific writing: knowledge of nursing students \\ Redacción científica: conocimiento de los estudiantes de enfermería
}

Recebido: 12/08/2021 | Revisado: 21/08/2021 | Aceito: 22/08/2021 | Publicado: 24/08/2021

Ruth Cristini Torres

ORCID: https://orcid.org/0000-0002-8664-192X Instituto de Hematologia e Hemoterapia de Sergipe, Brasil E-mail: ruthcristini@gmail.com

Letícia Tawane de Jesus Santos ORCID: https://orcid.org/0000-0003-3775-2049 Centro Universitário Estácio de Sergipe, Brasil E-mail: leticialjj@hotmail.com

Lúcia Plácida de Menezes

ORCID: https://orcid.org/0000-0002-7424-4580 Centro Universitário Estácio de Sergipe, Brasil E-mail: lup.dmenezes1605@yahoo.com

Marcelo Mendonça Mota

ORCID: https://orcid.org/0000-0003-1276-1942 Centro Universitário Estácio de Sergipe, Brasil

E-mail: mota.marcelo@gmail.com

Weber de Santana Teles

ORCID: https://orcid.org/0000-0003-1770-8278

Centro de Hemoterapia de Sergipe, Brasil

E-mail: arteecura@hotmail.com

Max Cruz da Silva

ORCID: https://orcid.org/0000-0002-6944-5986

Faculdade Pio Décimo, Brasil

E-mail: maxlfi@hotmail.com

Marcel Vinícius Cunha Azevedo

ORCID: https://orcid.org/0000-0002-5312-3333

Centro Universitário Estácio Sergipe, Brasil

E-mail: marcelvinicius49@gmail.com

Alejandra Debbo

ORCID: https://orcid.org/0000-0002-7743-5921

Universidade Tiradentes, Brasil

E-mail: aledebbo@hotmail.com

Maria Hozana Santos Silva

ORCID: https://orcid.org/0000-0001-5742-5366

Faculdade Ages de Medicina, Brasil

E-mail: hosana p@hotmail.com

Ana Fátima Souza Melo de Andrade

ORCID: https://orcid.org/0000-0002-7024-6175

Centro Universitário Estácio de Sergipe, Brasil

E-mail: anafatimamelo@hotmail.com

Paulo Celso Curvelo Santos Junior

ORCID: https://orcid.org/0000-0001-5834-6782

Universidade Tiradentes, Brasil

E-mail: paulo.curvelo.jr@gmail.com

Taíssa Alice Soledade Calasans

ORCID: https://orcid.org/0000-0003-0460-4437

Universidade Tiradentes, Brasil

E-mail: taissa.asc@gmail.com

Ângela Maria Melo Sá Barros

ORCID: https://orcid.org/0000-0003-4087-3247

Universidade Federal do Rio de Janeiro, Brasil

E-mail: angelsamelo@hotmail.com

\section{Resumo}

A cognição é algo intrínseco ao ser humano, e se desenrola por um encadeamento de etapas que discordam do tipo de aprendizado, que podem ser referentes a religião, filosofia, populares e artísticos. O processo de leitura e escrita são atividades presentes na vivência acadêmica, através dessas origina-se o conhecimento das práticas da futura carreira do universitário. Objetiva-se avaliar o nível de compreensão dos discentes a tratar sobre a escrita cientifica. Trata-se de uma pesquisa descritiva, com abordagem quantitativa, realizada em uma instituição de ensino superior do estado de 
Sergipe. A análise de dados foi realizada por meio dos programas Statistical Package For the Social Sciences for Windows - SPSS versão 21 e Microsoft Excel 2010 e distribuídos em frequências absoluta e relativa. Constatou-se que apenas 7,05\% dos pesquisados, de um total de 170 acadêmicos, apresentaram conhecimento sobre o a escrita científica. Por fim, discute-se a oferta da disciplina online e o papel dos docentes no desenvolvimento do conhecimento científicocrítico dos discentes.

Palavras-chave: Pesquisa científica e desenvolvimento tecnológico; Indicadores de produção científica; Pesquisa metodológica em enfermagem; Educação em saúde.

\begin{abstract}
Cognition is something intrinsic to human beings, and it unfolds through a chain of stages that disagree with the type of learning, which can be related to religion, philosophy, popular and artistic. The process of reading and writing are activities present in the academic experience, through which the knowledge of the practices of the university's future career originates. The objective is to assess the level of understanding of students to deal with scientific writing. This is a descriptive research, with a quantitative approach, carried out in a higher education institution in the state of Sergipe. Data analysis was performed using the Statistical Package for Social Sciences for Windows - SPSS version 21 and Microsoft Excel 2010 and distributed in absolute and relative frequencies. It was found that only $7.05 \%$ of respondents, out of a total of 170 academics, had knowledge about scientific writing. Finally, the offer of the online discipline and the role of teachers in the development of scientific-critical knowledge of students are discussed.

Keywords: Scientific research and technological development; Scientific production indicators; Methodological research in nursing; Health education.
\end{abstract}

\title{
Resumen
}

La cognición es algo intrínseco al ser humano, y se desarrolla a través de una cadena de etapas que no concuerdan con el tipo de aprendizaje, que puede estar relacionado con la religión, la filosofía, lo popular y lo artístico. El proceso de lectura y escritura son actividades presentes en la experiencia académica, a través de las cuales se origina el conocimiento de las prácticas de la futura carrera universitaria. El objetivo es evaluar el nivel de comprensión de los estudiantes para afrontar la redacción científica. Se trata de una investigación descriptiva, con enfoque cuantitativo, realizada en una institución de educación superior del estado de Sergipe. El análisis de los datos se realizó utilizando el Paquete Estadístico para Ciencias Sociales para Windows - SPSS versión 21 y Microsoft Excel 2010 y se distribuyó en frecuencias absolutas y relativas. Se encontró que solo el 7,05\% de los encuestados, de un total de 170 académicos, tenían conocimientos sobre redacción científica. Finalmente, se discute la oferta de la disciplina online y el papel de los docentes en el desarrollo del conocimiento científico-crítico de los estudiantes.

Palabras clave: Investigación científica y desarrollo tecnológico; Indicadores de producción científica; Investigación metodológica en enfermería; Educación para la salud.

\section{Introdução}

A geração de informações são os mais vultuosos aspectos de aditamento de uma corporação, sendo que o aprendizado de forma sistemática, arranjada, originado de estudos da ciência simboliza veículo de condensação de definido ramo de movimentação (Espuny, 2020).

A cognição é algo intrínseco ao ser humano, e se desenrola por um encadeamento de etapas que discordam do tipo de aprendizado, que podem ser referentes a religião, filosofia, populares e artísticos (Marconi, et al., 2016).

O processo de leitura e escrita são atividades presentes na vivência acadêmica, através dessas origina-se o conhecimento das práticas da futura carreira do universitário. A transcrição de todo o conhecimento produzido ao longo dos séculos, ou seja, a memória do saber científico só é possível por meio da escrita. A apropriação dessa herança vai além da compreensão da linguagem por meio transparente de propagação do conhecimento e requisita pensá-la em suas complexas dimensões e em seus efeitos na formação da identidade profissional do estudante (Litenski, et al.,2018).

A sapiência e o conhecimento da escrita científica se baseiam no setor chamado diacronia de corpus, em que os eventos da linguagem são investigados a partir da análise dos enormes volumes de textos (Osvaldo, 2014). O uso dos métodos de linguagem de corpus, gerou advertências importantes, sendo que a informação cientifica é enormemente tipificada, e um artigo tem uma estrutura praticamente fixa (Dutra, et al., 2010).

É vital considerar o comprometimento dos alunos como sendo fator indispensável para o início e progressão das ações indispensáveis à realização das tarefas da vida estudantil, claramente dependente do nível de desenvolvimento intelectual que 
cada discente apresentará na sua aprendizagem (Freitas, 2018). Acadêmicos são instigados a abarcar suas novas atribuições como parte importante da instituição universitária, incumbindo-se de zelar para sua "voz pessoal" ser ouvida no discurso e na escrita científica, refletindo sobre o uso inadequado dos textos alheios, a fim de evitar o plágio Bezerra (2015).

O aluno carece de praticar, essa metodologia pedagógica, saber criticar, ser hábil na aquisição de informações sobre diversos temas, que promoverá o alargamento do silogismo científico e, por conseguinte seu absorvimento (Figueiredo, et al., 2016).

No cenário atual, debates sobre o ensino científico (EC) têm se destacado em estudos no universo da educação em ciências. Compreendendo o ensino científico como método e a criança como tendente incorporado em um cenário social, passível de agência e cidadania, é válido pensar que a criança pode ser alfabetizada cientificamente (Marques \& Marandino, 2014).

Compartilhando da mesma ideia, (Carmo \& Franco, 2019) expuseram a relevância das competências que os docentes precisam buscar, bem como o domínio específico sobre determinada disciplina desde o âmbito didático-pedagógico, tecnológico, linguístico e social além da aquisição de saberes estratégicos de repasse desses conhecimentos, na capacidade de aliar inovações tecnológicas ao desenvolvimento das práticas educativas, na redação e interpretação de temas escritos possibilitando ao grupo acadêmico um meio dialógico e dialético e sob seu intermédio, clara compreensão para o construto de sua aprendizagem.

A Enfermagem cada vez mais vem ganhando espaço no campo da pesquisa científica, especialmente em relação ao aprimoramento técnico, metodológico e cientifico dos estudos produzidos por enfermeiros nos últimos anos (Gomes, 2017).

O Brasil tem se destacado, entre os países latino-americanos, no desenvolvimento de pesquisa cientifica em enfermagem e que essa área apresentou grandes avanços nos seus métodos de pesquisar e de educar, levando a mudanças no desenvolvimento curricular dos cursos de formação de nível superior (Backes, et al., 2012) Entretanto, averiguando as mais relevantes revistas de enfermagem nacionais, há evidências de que o modo qualitativo ainda é pouco estudado em pesquisas realizadas por enfermeiros brasileiros (Alencar, et al., 2018).

Diante do exposto, surgem as questões norteadoras deste estudo: Qual o nível de conhecimento dos acadêmicos de enfermagem sobre a escrita cientifica? Quais as dificuldades apresentadas pelos acadêmicos, diante da escrita cientifica? Qual o nível de conhecimento dos acadêmicos de enfermagem sobre escrita cientifica ao final do curso? Quais as sugestões dos acadêmicos acerca do ensino da escrita científica?

O presente estudo tem como hipótese o despreparo que os acadêmicos de enfermagem apresentam para desenvolver a escrita científica. Sendo assim, justifica-se pela crença dos autores de que essa escrita se corretamente redigida tem grande relevância por nortear a vida acadêmica. Devido ao fato de os estudantes de enfermagem não saberem produzir e fundamentar corretamente trabalhos acadêmicos, consideramos de suma importância abordar esse tema para contribuir para a melhoria do aprendizado dessa metodologia. Portanto, objetiva-se avaliar o nível de conhecimento dos acadêmicos de enfermagem sobre esse tipo escrita, especificamente.

\section{Metodologia}

Trata-se de uma pesquisa de caráter descritivo com abordagem quantitativa, realizada em uma Instituição de Ensino Superior (IES) do estado de Sergipe. Foram incluídos acadêmicos de enfermagem dos $5^{\circ}$ e $10^{\circ}$ períodos, que aceitaram participar da pesquisa. Foram excluídos aqueles que faltaram aula no dia da coleta de dados. Realizou-se um cálculo amostral (MIOT, 2011) com base na população de acadêmicos do $5^{\circ}$ período e do $10^{\circ}$ no ano de 2019 , resultando em uma amostra mínima de 70 sujeitos do $5^{\circ}$ período e 100 do $10^{\circ}$ período. 
Aplicou-se um roteiro de entrevista semiestruturado abrangendo a temática da escrita científica com o total de 10 questões. A coleta de dados foi realizada por meio de agendamento prévio nas turmas de $5^{\circ}$ e $10^{\circ}$ períodos nos dias de aula teórica.

Esta pesquisa aprovada pelo Comitê de Ética em Pesquisa (CEP) da Estácio de Sergipe, conforme a resolução $n^{\circ}$ 466/2012 do Conselho Nacional de Saúde, sob nº de CAAE: 21190119.5.0000.8079 e parecer de aprovação $n^{\circ} 3.601 .582$. Todos os participantes do estudo foram convidados a assinar o Termo de Consentimento Livre e Esclarecido (TCLE).

Os dados foram analisados por meio dos programas Statistical Package For the Social Sciences for Windows - SPSS versão 21 e Microsoft Excel 2010 e distribuídos em frequências absolutas e relativas.

\section{Resultados}

Nesta pesquisa participaram 170 acadêmicos do curso de enfermagem sendo 100 alunos do $10^{\circ}$ período e 70 do $5^{\circ}$ período com faixa etária predominante de 23 a 28 anos. As respostas dos estudantes referentes ao aproveitamento da matéria metodologia científica na modalidade on-line foram avaliadas em 3 categorias: Conteúdo passado de forma clara, dúvidas esclarecidas e se o conteúdo abordado foi suficiente para desenvolver as atividades acadêmicas.

Ao serem questionados sobre se o conteúdo foi ministrado de forma clara, destacaram-se $83(49 \%)$ relatos de conteúdo regular e em relação aos questionamentos sobre dúvidas esclarecidas, 87 (51,2\%) universitários consideraram regular. Quando indagados a respeito do conteúdo ser suficiente para realização de suas atividades, 77 (45,3\%) responderam que foi regular (Figura 1).

Figura 1 - Distribuição dos universitários referente aos relatos sobre o aproveitamento da matéria metodologia científica na modalidade on-line. Aracaju, Sergipe, 2019.

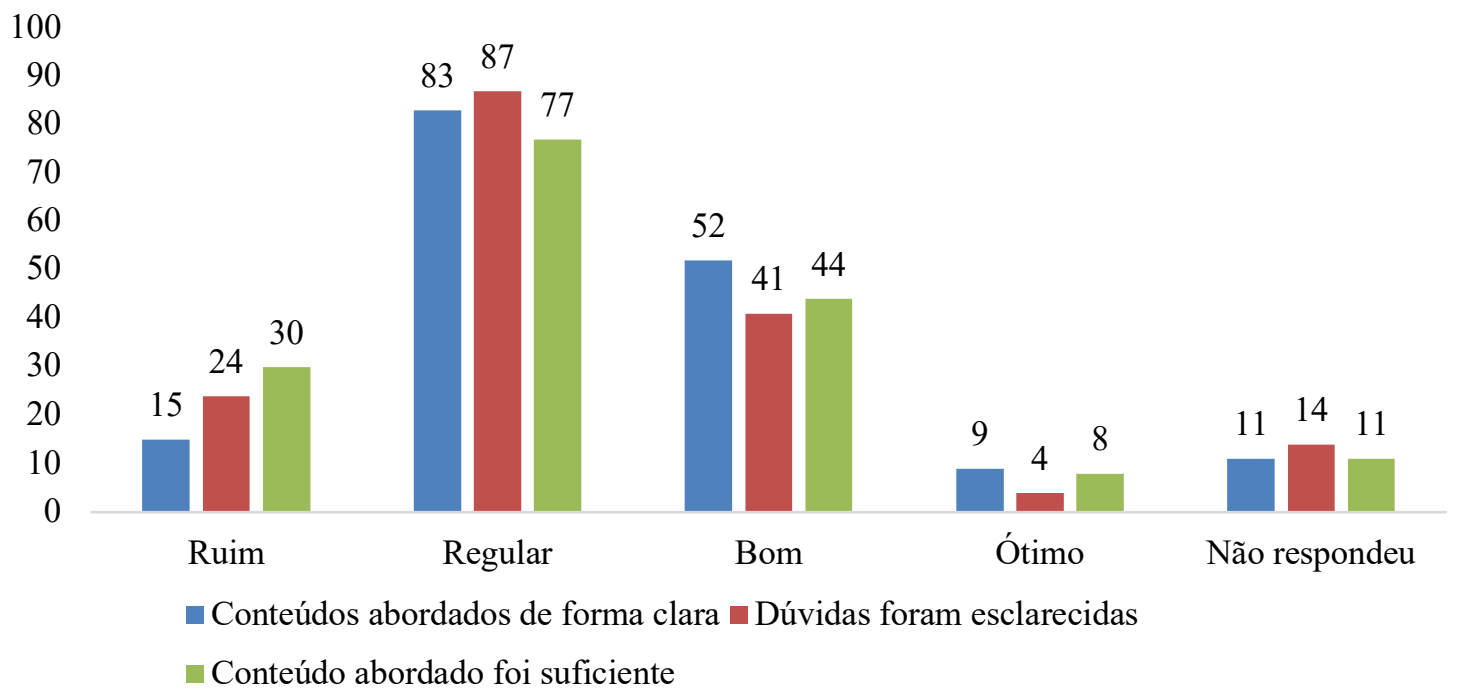

Fonte: Autores.

Percebeu-se o predomínio de 83 (48,8\%) entrevistados que classificaram como regular a transmissão clara do conteúdo, contrastando com $9(5,2 \%)$ que consideraram como ótimo. Já na categoria “dúvidas esclarecidas”, 87 (51,1\%) responderam que foi regular e apenas 4 (2,3\%) relataram ótimo. Vale ressaltar que esta parte da pesquisa não segregou alunos do $5^{\circ}$ e $10^{\circ}$ períodos.

Realizou-se a distribuição dos acadêmicos em relação a opinião sobre a modalidade da oferta da disciplina de metodologia, onde os acadêmicos dos $5^{\circ}$ e $10^{\circ}$ períodos pontuaram sobre a satisfação com a oferta da disciplina on-line. 
Constatou-se que $120(70,6 \%)$ dos entrevistados acreditam que a oferta na modalidade on-line da disciplina de metodologia científica não atende às suas reais necessidades (Figura 2).

Figura 2 - Distribuição dos acadêmicos em relação a opinião sobre a modalidade da oferta da disciplina de metodologia científica. Aracaju, Sergipe, 2019.

A oferta on-line atende às minhas necessidades

A oferta on-line atende parcialmente às minhas necessidades

A oferta on-line não atende às minhas necessidades

\section{$7(4,1 \%)$}

$43(25,3 \%)$

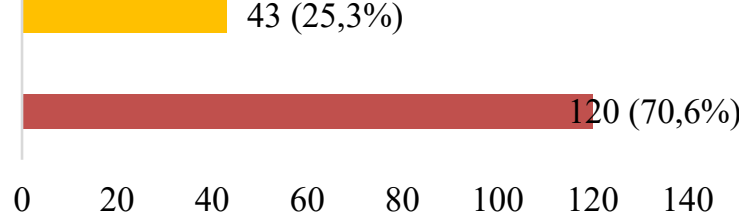

Fonte: Autores.

Ao verificar a distribuição de universitários em relação a alegação sobre os docentes ensinarem como escrever trabalhos científicos em outras disciplinas, as respostas dos discentes de enfermagem foram separadas entre $5^{\circ}$ e $10^{\circ}$ períodos. Entre as turmas, tanto os $53(75,7 \%)$ universitários do $5^{\circ}$ período, quanto os $65(65 \%)$ do $10^{\circ}$ período, responderam "Não" (Figura 3).

Figura 3 - Distribuição de universitários em relação a alegação sobre os docentes ensinarem como escrever trabalhos científicos em outras disciplinas. Aracaju, Sergipe, 2019.

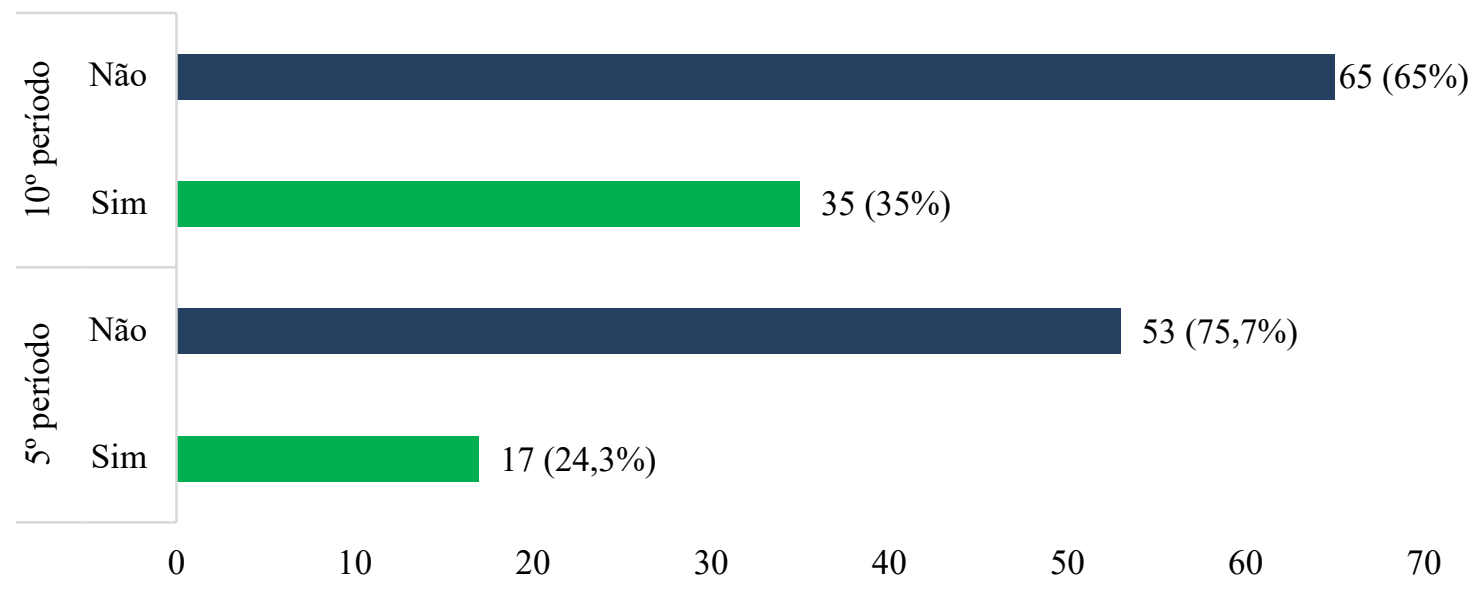

Fonte: Autores.

Em relação ao conhecimento acerca da aplicação das normas da ABNT, os dados foram analisados separando-se os universitários do $5^{\circ}$ e $10^{\circ}$ períodos. Dentre os alunos do $5^{\circ}$ período, 51 responderam que não sabem aplicar adequadamente as normas da ABNT e dos alunos entrevistados no $10^{\circ}$ período, 66 alegaram saber aplicar as normas (Figura 4), o que demonstra uma expressiva melhora desse conhecimento ao final do curso de graduação. 
Figura 4 - Distribuição de universitários sobre o conhecimento acerca da aplicação das normas da ABNT. Aracaju, Sergipe, 2019.

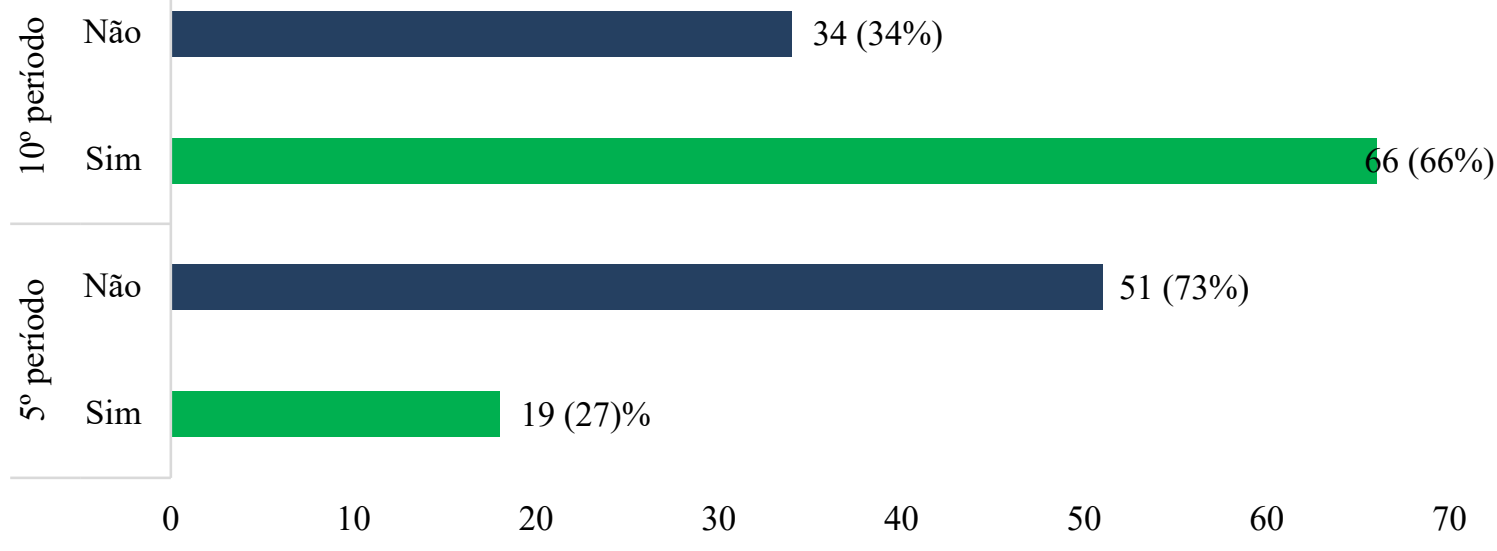

Fonte: Autores.

Tendo sido questionados aos acadêmicos sobre o que eles entendiam por "Escrita científica", visando saber o nível de compreensão dos mesmos a respeito dessa temática, as respostas foram classificadas em: satisfatória (7\%), parcialmente satisfatória $(26,5 \%)$ e não satisfatória $(66,5 \%)$.

\section{Discussão}

Os dados coletados evidenciam a necessidade de haver maior ênfase na abordagem do conceito da escrita técnica, urge que seja dispensado uma atenção diferenciada o mais precocemente possível em todos os períodos do curso de graduação em enfermagem para que seja fomentado nos acadêmicos o interesse aguçado sobre o conhecimento científico, possibilitando aos mesmos um saber refinado, visando o aprimoramento das produções acadêmico-científicas.

Considerando que as normas da ABNT são ferramentas de grande relevância no mundo acadêmico e no processo da escrita científica, a maioria dos alunos do $5^{\circ}$ e $10^{\circ}$ períodos referiram que os docentes de outras disciplinas não ensinam sobre a escrita científica, revelando que ao longo do curso não houvera avanço significativo da disposição dos professores em ensiná-la melhor.

Estudos comprovaram que os pesquisados atribuíram o sucesso da produção do saber científico ao fato do professortutor portar domínio da disciplina ensinada, das tecnologias de informação e comunicação (TIC) e da interpelação ao aluno em educação à distância (Carmo \& Franco, 2019).

Destaca-se que a escrita científica é alicerce fundamental para que se possa construir através do discurso e da interação com outros pensadores, uma identidade marcante no meio acadêmico-científico, caracterizando que a escrita científica deve ser aprendida e executada pelo estudante, por estar intrinsecamente ligada à formação discursiva de sua personalidade acadêmica (Bezerra, 2015).

Porém notou-se que a maioria dos alunos do $10^{\circ}$ período acreditam saber aplicar as normas da ABNT, enquanto que a maioria dos alunos do $5^{\circ}$, afirmam não saber dimensiona-las. Quanto ao entendimento dos discentes acerca da escrita cientifica e das normas da ABNT, os dados colhidos entre ambos períodos, evidenciam que apenas a minoria dos pesquisados demonstraram conhecê-las adequadamente. 
Em um estudo realizado com estudantes, ressaltaram em relação às particularidades das normas do linguajar científico, todos os pesquisados indicaram como dificuldade usual na escrita técnica, a imposição constante de elaborar referências e meios de citação, os quais os entrevistados alegaram não terem tido vivências suficiente (Litensk \& Pan, 2018).

O saber da escrita científica deverá ser comprovado na execução das tarefas acadêmicas, por meio de avaliações que correlacionem o rendimento escolar com a construção da escrita (Festas, et al., 2018)

Além dos estudos científicos e dos métodos de aprendizagem, a ampliação do saber técnico também tem relação com as ações de extensão promovidas pelas universidades, favorecendo a integração ensino, pesquisa e prática (Forfproex, 2012). Comumente a inclusão de proposta de extensão promove a população e ao aluno a experimentação e o desenvolvimento de práticas observadas e analisadas, no setor teórico o que auxiliará na consolidação do seu aprendizado (Nascimento, 2012).

Nessa contextualização da soberania do aluno da área de saúde aparece métodos de ensino e aprendizagem com um novo parecer educativo em que o candidato a sapiência se torna consciente de sua experiência (O'Connor, et al., 2004). Essa metodologia gera inúmeras possibilidades de desenvolver habilidades acerca dos conteúdos teóricos vivenciados em sala de aula, engenhosidade para os enfretamentos da profissão e incitamento ao desenvolvimento de pesquisas (Sobral, et al., 2012). Por outro lado, contrapartida alunos e profissionais que não vivenciaram a prática científica, podem ser mais predispostos a não considerar conjunturas diárias da sua profissão (Basnet, et al., 2014).

Evidenciou-se no presente estudo uma grande deficiência na estratégia de ensino dessa metodologia aos acadêmicos de enfermagem, deixando à mostra um certo desinteresse dos docentes a fim de melhor contribuir para seu ensino.

Neste contexto, as oficinas acadêmicas se revelaram como estratégia viável de formação profissional, focando na desnaturalização das dificuldades dos estudantes entendidas como (in) competências individuais, propiciando um espaço para os acadêmicos se posicionarem diante dos textos que leem, orientando-os em suas matrizes discursivas, expondo seus sentimentos e obstáculos diante das excessivas tarefas exigidas, explicando como fazer, por exemplo, uma resenha, um relatório, ou mesmo, uma citação, a paráfrase, a condensação (Litensk \& Pan, 2018).

\section{Conclusão}

Após uma análise crítica das circunstâncias que envolvem a disciplina de metodologia e seu aprendizado, sugere-se que tal disciplina em sua oferta on-line não contribui satisfatoriamente para que os alunos sejam capazes de construir trabalhos científicos que respondam à demanda acadêmica e com qualidade científica.

Neste aspecto, os docentes também têm papel fundamental, uma vez que estes devem orientar e guiar o aluno desde seu início na graduação. Portanto, faz-se necessário que as instituições oportunizem a qualificação de seus profissionais. Fora notado pouco interesse em capacitar os acadêmicos de enfermagem para a produção científica.

Esta pesquisa tem a expectativa de que, partindo dos resultados expostos, possam ser encontradas as melhores formas e alternativas de sanar a deficiência existente dos acadêmicos na construção do saber científico e, criar uma melhor metodologia para os futuros alunos, afim de que estes sejam mais envolvidos com o mundo científico e se sintam incentivados e preparados para realizar pesquisas científicas.

\section{Referências}

Alencar, A. M. P. G., et. al. (2018). Descrição interpretativa: Uma abordagem metodológica viável para a pesquisa em enfermagem. Escola Anna Nery. 22(3), $1-8$.

Basnet, B. \& Bhandari, A. (2014). Investing in medical student's research: promoting future of evidence based medicine in Nepal. Health Renaissance. 11(3), 297-300.

Backes, V. M. S., et al. (2012). Grupos de Pesquisa de Educação em Enfermagem do Brasil. Revista da Escola de Enfermagem da USP. 46(2), 436-442. 
Bezerra, B. (2015). Letramentos acadêmicos e construção da identidade: a produção do artigo científico por alunos de graduação. Linguagem em Discurso. Santa Catarina. 15(1), 61-76.

Carmo, R. O. S. \& Franco, A. P. (2019). Da Docência Presencial à Docência Online: Aprendizagens de Professores Universitários na Educação à Distância. Educação em Revista. 3, 1-15.

Dutra, D. P. \& Silero, R. P. (2010). Descobertas linguísticas para pesquisadores e aprendizes: a linguística de Corpus e o ensino de gramática. Revista Brasileira de Linguística Aplicada, 10(4), 909-930.

Espuny, H. G. (2020). A Metodologia de Pesquisa Científica como Ferramenta de Produção de Conhecimento na Polícia Civil. Texto baseado em monografia apresentada à Banca de Seleção de Professor Temporário de Metodologia da Pesquisa Científica, na Academia de polícia Doutor Coriolano Nogueira Cobra, 2020 .

Festas, M. I., et al. (2018). Envolvimento, desempenho acadêmico e composição escrita. Educação e Pesquisa. 44, 1-17.

Fórum de Pró-Reitores de Extensão das Universidades Públicas Brasileiras. Política nacional de extensão universitária, FORFPROEX.

Nascimento, I. R. T. (2012). A indissociabilidade entre pesquisa e extensão nas universidades: o caso da ITES/UFBA. Rev NAU Soc. 3(5), 41-6.

Figueiredo, W. P. S., Moura, N. P. R. \& Tanajura, D. M. (2016). Ações de pesquisa e extensão e atitudes científicas de estudantes da área da saúde. Arq. Ciênc. Saúde.

Gomes, A. T. L., Salvador, P. T. C. O., et. al. (2017). Os caminhos percorridos pela Enfermagem brasileira na pesquisa: Estudo documental. Online Brazilian Journal of Nursing. 16(2), 226-232.

Litenski, A. C. L. \& Pan, M. A. G. S. (2018). Letramentos e identidade profissional: reflexões sobre leitura, escrita e subjetividade na universidade. Psicologia Escolar e Educacional, 22(3), 527-534.

Marconi, M. A. \& Lakatos, E. M. (2016). Fundamentos de Metodologia Científica. (7a ed.), Atlas.

Marques, A. C. T. L. \& Marandino, M.(2014). Alfabetização científica ou letramento científico: interesses envolvidos nas interpretações da noção de scietific literacy. Educação e Pesquisa. 44, 169-186.

O’Connor, J. P. B. \& Kanga, D. R. J. (2004). Academic medicine: time for reinvention: medical education, training, and research are under threat because academic medicine is undervalued. BMJ. 328(7430), 45-6.

Miot, H. A. (2011). Tamanho da amostra em estudos clínicos e experimentais. J. Vasc. Bras. 10(44), 275-278.

Osvaldo, N. \& Oliveira, Jr. (2014). As Linguagens do Conhecimento. Editora Cubo.

Sobral, F. R. \& Campos, C. J. G. (2012). Utilização de metodologia ativa no ensino e assistência de enfermagem na produção nacional: revisão integrativa. Rev Esc Enferm USP. 46(1), 208-18

Vizeu, F., Macadar, M. A. \& Graeml, A. R. (2016). Produtivismo acadêmico baseado em uma perspectiva habermasiana. Caderno EBAPE. BR. 14(4), 9841000 